\title{
UNIFORM CHERNOFF AND \\ DVORETZKY-KIEFER-WOLFOWITZ-TYPE INEQUALITIES FOR MARKOV CHAINS AND RELATED PROCESSES
}

\author{
ARYEH KONTOROVICH ${ }^{* * *}$ AND \\ ROI WEISS, ${ }^{*}$ Ben-Gurion University of the Negev
}

\begin{abstract}
We observe that the technique of Markov contraction can be used to establish measure concentration for a broad class of noncontracting chains. In particular, geometric ergodicity provides a simple and versatile framework. This leads to a short, elementary proof of a general concentration inequality for Markov and hidden Markov chains, which supersedes some of the known results and easily extends to other processes such as Markov trees. As applications, we provide a Dvoretzky-Kiefer-Wolfowitz-type inequality and a uniform Chernoff bound. All of our bounds are dimension-free and hold for countably infinite state spaces.
\end{abstract}

Keywords: Concentration of measure; Markov chain; hidden Markov chain; Chernoff; Dvoretzky-Kiefer-Wolfowitz

2010 Mathematics Subject Classification: Primary 60E15

Secondary $60 \mathrm{~J} 10$

\section{Introduction}

\subsection{Background}

The last two decades have seen a flurry of activity in concentration of measure for nonindependent processes. A recent survey may be found in [19], with pointers to more specialized surveys therein. Rather than recapitulating these surveys here, we shall proceed directly to the relevant recent developments. Let $X_{1}, X_{2}, \ldots$ be a sequence of $\mathbb{N}$-valued random variables obeying some joint law (distribution). Using the shorthand $\mathcal{L}\left(X_{j}^{n} \mid X_{1}^{i}=x\right)$ to denote the law of $\left(X_{j}, \ldots, X_{n}\right)$ conditioned on $\left(X_{1}, \ldots, X_{i}\right)=x \in \mathbb{N}^{i}$, let us define, for $n \in \mathbb{N}, 1 \leq i<j \leq n$, $y \in \mathbb{N}^{i-1}$ and $w, w^{\prime} \in \mathbb{N}$,

$$
\eta_{i j}\left(y, w, w^{\prime}\right)=\left\|\mathcal{L}\left(X_{j}^{n} \mid X_{1}^{i}=y w\right)-\mathcal{L}\left(X_{j}^{n} \mid X_{1}^{i}=y w^{\prime}\right)\right\|_{\mathrm{TV}},
$$

(where $\|\cdot\|_{\mathrm{TV}}=\frac{1}{2}\|\cdot\|_{1}$ is the total variation norm) and

$$
\bar{\eta}_{i j}=\sup _{y \in \mathbb{N}^{i-1}, w, w^{\prime} \in \mathbb{N}} \eta_{i j}\left(y, w, w^{\prime}\right)
$$

The coefficients $\bar{\eta}_{i j}$, termed $\eta$-mixing coefficients in [21], play a central role in several recent concentration results. Define $\Delta$ to be the upper-triangular $n \times n$ matrix, with $\Delta_{i i}=1$ and $\Delta_{i j}=\bar{\eta}_{i j}$ for $1 \leq i<j \leq n$.

Received 9 January 2013; revision received 14 January 2014.

* Postal address: Department of Computer Science, Ben-Gurion University, Beer Sheva, 84105, Israel.

** Email address: karyeh@cs.bgu.ac.il 
In 2007, [8] and [21] independently proved that for any $f: \mathbb{N}^{n} \rightarrow \mathbb{R}$ with $\|f\|_{\text {Lip }} \leq 1$ with respect to the Hamming metric meaning: if $x, y \in \mathbb{N}^{n}$ differ in only one coordinate then $|f(x)-f(y)| \leq 1$, see Section 2.7), we have

$$
P(|f-\mathbb{E} f|>n \varepsilon) \leq 2 \exp \left(-\frac{2 n \varepsilon^{2}}{\min \left\{\|\Delta\|_{2},\|\Delta\|_{\infty}\right\}^{2}}\right),
$$

where $\|\Delta\|_{p}$ is the $\ell_{p}$ operator norm ([8] achieved the better constant in the exponent, given here). Earlier, Samson [33] had given a concentration result for convex $\ell_{2}$-Lipschitz functions $f:[0,1]^{n} \rightarrow \mathbb{R}$, which likewise involved the coefficients $\bar{\eta}_{i j}$, and these are also implicit in Marton's earlier work [27], [28], and [29].

In order to apply (1.2) in a Markov setting, we must upper-bound $\|\Delta\|_{2}$ or $\|\Delta\|_{\infty}$ for the Markov chain in question. The earliest such results relied on contraction. Let $p(\cdot \mid \cdot)$ be the transition kernel associated with a given Markov chain, and define the (Döblin) contraction coefficient

$$
\kappa=\sup _{x, x^{\prime} \in \mathbb{N}}\left\|p(\cdot \mid x)-p\left(\cdot \mid x^{\prime}\right)\right\|_{\mathrm{TV}}
$$

It is shown in [21] and [33] that $\bar{\eta}_{i j} \leq \kappa^{j-i}$ and, therefore, $\|\Delta\|_{\infty} \leq(1-\kappa)^{-1}$; this implies the concentration bound

$$
\mathbb{P}(|f-\mathbb{E} f|>n \varepsilon) \leq 2 \exp \left(-2(1-\kappa)^{2} n \varepsilon^{2}\right)
$$

for 1-Lipschitz functions $f$, which Marton [26] had essentially obtained earlier by other means.

The contraction method was pushed further to obtain concentration results for hidden Markov chains [21], undirected Markov chains and Markov tree processes [19], but its applicability requires the rather stringent condition that $\kappa<1$. Marton [27] had already observed that a significantly weaker mixing condition suffices, and yields tighter and more informative bounds. Indeed, consider a Markov chain with stationary distribution $\pi$ and conditional $s$ th step distribution $\mathcal{L}\left(X_{s} \mid X_{1}=x\right)$, and define the 'inverse mixing time'. (This terminology is nonstandard.)

$$
\tau_{s}=\sup _{x \in \mathbb{N}}\left\|\mathcal{L}\left(X_{s} \mid X_{1}=x\right)-\pi\right\|_{\mathrm{TV}}
$$

A simple calculation (Lemma 2.3) shows that $\bar{\eta}_{i j} \leq 2 \tau_{j-i}$, and, thus,

$$
\|\Delta\|_{\infty}-1=\max _{1<i<n} \sum_{j=i+1}^{n} \bar{\eta}_{i j} \leq 2 \max _{1<i<n} \sum_{j=i+1}^{n} \tau_{j-i} .
$$

A rich body of work deals with bounding $\tau_{s}$ via spectral [15], Poincaré [11], log-Sobolev [10], and Lyapunov [22] methods, among others (and the references therein). From our perspective, the geometric ergodicity condition allows for the simplest exposition while sacrificing the least generality. A Markov chain is said to be geometrically ergodic with constants $1 \leq G<\infty$ and $0 \leq \theta<1$ if

$$
\tau_{s} \leq G \theta^{s-1}, \quad s=1,2, \ldots
$$

Any finite ergodic Markov chain is geometrically ergodic, and the dependence of $G, \theta$ on various structural properties of the chain in question is the subject of a diverse and prolific literature (including the references above). We also stress that the geometric ergodicity assumption is largely dictated by expositional convenience, since any nontrivial bound on the inverse mixing time $\tau_{s}$ will yield straightforward analogues of our results. 
In this paper we explore some consequences of geometric ergodicity as pertaining to concentration and statistical inference for Markov and hidden Markov chains. We leverage two basic insights: (i) even though hidden Markov chains are a considerably richer class of processes than Markov chains (there exist HMMs not realizable by any finite-order Markov chain), for the purposes of measure concentration, the underlying Markov chain is all that matters, and (ii) geometric ergodicity, while significantly more general than contractivity, yields essentially the same concentration bounds. Another advantage of our approach is its elementary nature: taking the bound in (1.2) as a given, nothing beyond basic linear algebra is used.

Given the recent interest in prediction and parameter inference for HMMs [3], [17], [20], [31], [34], [36], our results have the potential to be applicable beyond the abstract setting studied here. Furthermore, since concentration results for Markov chains extend easily for other Markov-type processes (such as trees [19]), our results here should extend to those as well.

\subsection{Main results}

Concentration. Our first result is a concentration inequality for hidden Markov chains, which generalizes many of the previous bounds. Henceforth, we will write ' $(G, \theta)$-geometrically ergodic' as shorthand for 'geometrically ergodic with constants $1 \leq G<\infty$ and $0 \leq \theta<1$ '. Hidden Markov chains and their associated notions of stationarity and geometric ergodicity are formally defined in Section 2.1.

Theorem 1.1. Let $Y_{1}, Y_{2}$, . be an $\mathbb{N}$-valued hidden Markov chain whose underlying $\mathbb{N}$-valued Markov chain is $(G, \theta)$-geometrically ergodic. Then, for any $n \in \mathbb{N}$ and $f: \mathbb{N}^{n} \rightarrow \mathbb{R}$ with $\|f\|_{\text {Lip }} \leq 1$ under the Hamming metric (see Section 2.7), we have

$$
\mathbb{P}\left(f\left(Y_{1}^{n}\right)-\mathbb{E} f\left(Y_{1}^{n}\right)>n \varepsilon\right) \leq \exp \left(-\frac{n(1-\theta)^{2} \varepsilon^{2}}{2 G^{2}}\right),
$$

with an identical bound for the other tail.

Although the result in Theorem 1.1 does not appear to have been published anywhere, it is a simple consequence of widely known facts (we give a proof in Section 2 for completeness). Our main contribution lies in the apparently novel applications.

Dvoretzky-Kiefer-Wolfowitz-type inequality. Let us recall the Dvoretzky-Kiefer-Wolfowitz (DKW) inequality [14], [30], stated here for the discrete case. Suppose $X_{1}, X_{2}, \ldots$ are independent, identically distributed (i.i.d.) $\mathbb{N}$-valued random variables with common distribution function $F$, we define the empirical distribution function $\hat{F}_{n}$ induced by $\left(X_{1}, \ldots, X_{n}\right)$ as

$$
\hat{F}_{n}(x)=\frac{1}{n} \sum_{i=1}^{n} \mathbf{1}_{\left\{X_{i} \leq x\right\}}, \quad x \in \mathbb{N} .
$$

The DKW inequality states that

$$
\mathbb{P}\left(\sup _{x \in \mathbb{N}}\left|\hat{F}_{n}(x)-F(x)\right|>\varepsilon\right) \leq 2 \exp \left(-2 n \varepsilon^{2}\right), \quad \varepsilon>0, n \in \mathbb{N} .
$$

We present the following Markovian version of this inequality.

Theorem 1.2. Let $Y_{1}, Y_{2}, \ldots$ be a stationary $\mathbb{N}$-valued $(G, \theta)$-geometrically ergodic Markov or hidden Markov chain with stationary distribution $\rho \in \mathbb{R}^{\mathbb{N}}$. For $n \in \mathbb{N}$, define $\hat{\rho}^{(n)} \in \mathbb{R}^{\mathbb{N}}$ to 
be the empirical estimate of $\rho$

Then

$$
\hat{\rho}_{y}^{(n)}=\frac{1}{n} \sum_{i=1}^{n} \mathbf{1}_{\left\{Y_{i}=y\right\}}, \quad y \in \mathbb{N} .
$$

$$
\mathbb{P}\left(\left\|\rho-\hat{\rho}^{(n)}\right\|_{\infty}>\sqrt{\frac{1+2 G \theta}{n(1-\theta)}}+\varepsilon\right) \leq \exp \left(-\frac{n(1-\theta)^{2} \varepsilon^{2}}{2 G^{2}}\right), \quad n \in \mathbb{N}, \varepsilon>0 .
$$

As we show in Section 2.6, the assumption that the chain starts in the stationary distribution is not at all restrictive. Note that a naive application of Theorem 1.1 to each $\hat{\rho}_{y}^{(n)}$ individually, combined with the union bound, would yield

$$
\mathbb{P}\left(\left\|\rho-\hat{\rho}^{(n)}\right\|_{\infty}>\varepsilon\right) \leq 2\|\rho\|_{0} \exp \left(-\frac{n(1-\theta)^{2} \varepsilon^{2}}{2 G^{2}}\right),
$$

where $\|\rho\|_{0}$ is the number of nonzero entries in $\rho$. The bound in (1.6) is considerably weaker than the one in Theorem 1.2 and in particular is vacuous for $\rho$ with infinite support.

Uniform Chernoff bound. Let $Y_{1}, Y_{2}, \ldots$ be a stationary $\mathbb{N}$-valued $(G, \theta)$-geometrically ergodic Markov or hidden Markov chain as above, and consider the occupation frequency

$$
\hat{\rho}^{(n)}(E)=\frac{1}{n} \sum_{i=1}^{n} \mathbf{1}_{\left\{Y_{i} \in E\right\}}, \quad E \subseteq \mathbb{N} .
$$

A naive application of Theorem 1.1 might yield a deviation bound along the lines of

$$
\mathbb{P}\left(\left|\rho(E)-\hat{\rho}^{(n)}(E)\right|>\varepsilon\right) \leq 2|E| \exp \left(-\frac{n(1-\theta)^{2} \varepsilon^{2}}{2|E|^{2} G^{2}}\right),
$$

where $|E|$ is the cardinality of $E$ and $\rho$ is the stationary distribution as above. We will give a much stronger bound, that is not only independent of $E$ but is actually uniform over all $E \subseteq \mathbb{N}$.

Theorem 1.3. For stationary $(G, \theta)$-geometrically ergodic (hidden) Markov chains, define

$$
\Lambda_{n}(\rho)=\gamma_{n}(G, \theta) \sum_{\rho_{y} \geq 1 / n} \sqrt{\rho_{y}}+\min \left\{\gamma_{n}(G, \theta) \sum_{\rho_{y}<1 / n} \sqrt{\rho_{y}}, \sum_{\rho_{y}<1 / n} \rho_{y}\right\}, \quad n \in \mathbb{N},
$$

where

Then

$$
\gamma_{n}(G, \theta)=\frac{1}{2} \sqrt{\frac{1+2 G \theta}{n(1-\theta)}}
$$

(a) for all distributions $\rho \in \mathbb{R}^{\mathbb{N}}, \lim _{n \rightarrow \infty} \Lambda_{n}(\rho)=0$,

(b) $\mathbb{P}\left(\sup _{E \subseteq \mathbb{N}}\left|\rho(E)-\hat{\rho}^{(n)}(E)\right|>\Lambda_{n}(\rho)+\varepsilon\right) \leq \exp \left(-n(1-\theta)^{2} \varepsilon^{2} / 2 G^{2}\right)$.

We remark that although $\Lambda_{n}(\rho) \rightarrow 0$ as $n \rightarrow \infty$ for all stationary distributions $\rho$, the rate of decay is $\rho$-dependent and may be arbitrarily slow for heavy-tailed distributions (compare [4, Lemma 8]).

For $\rho$ satisfying $\sum_{y \in \mathbb{N}} \sqrt{\rho_{y}}<\infty$, the bound in Theorem 1.3 may be somewhat simplified via

$$
\Lambda_{n}(\rho) \leq \gamma_{n}(G, \theta) \sum_{y \in \mathbb{N}} \sqrt{\rho_{y}} .
$$

As with Theorem 1.2, the stationarity assumption $p_{1}=\pi$ is not very restrictive (see Section 2.6). 


\subsection{Related work}

In parallel to the work on concentration of measure results for Markov chains ([1], [2], [7], [21], [26], [33]), grew a body of work on Chernoff-type bounds for these processes. The papers [12], [13], [16], [18], [24] played a founding role, and various extensions and refinements followed [23], [35]. In a remarkable recent development [9], optimal Chernoff-Hoeffding bounds are obtained based on the mixing time at a constant threshold.

Concentration of Lipschitz functions of mixing sequences, with applications to the Kolmogorov-Smirnov statistic, were considered in [32]. Bobkov and Götze [5] examined the concentration of empirical distributions for nonindependent sequences satisfying Poincaré or log-Sobolev inequalities.

\section{Methods and proofs}

\subsection{Preliminaries}

For clarity, we will sometimes write the matrix entry $A_{x, y}$ as $A(x \mid y)$. We will use the terms hidden Markov chain and HMM interchangeably.

Markov chains. We will represent Markov kernels by column-stochastic $\mathbb{N} \times \mathbb{N}$ matrices denoted by the letter $A$. Thus, a Markov chain with transition kernel $A$ and initial distribution $p_{1}$ induces the following distribution on $\mathbb{N}^{n}$

$$
\mathcal{L}\left(X_{1}, \ldots, X_{n}\right)=p_{1}\left(X_{1}\right) \prod_{i=1}^{n-1} A\left(X_{i+1} \mid X_{i}\right)
$$

Hidden Markov chain. A hidden Markov chain (also known as hidden Markov model (HMM)) is specified by the triple $\left(p_{1}, A, B\right)$, where $\left(p_{1}, A\right)$ are the Markov chain parameters, as above, and $B$ is an $\mathbb{N} \times \mathbb{N}$ column-stochastic matrix of emission probabilities. This HMM induces a distribution on $\mathbb{N}^{n}$ as follows. Let $X \in \mathbb{N}^{n}$ be distributed according to (2.1) and define the conditional distribution $\mathcal{L}(\cdot \mid X)$ over $Y \in \mathbb{N}^{n}$ as

$$
\mathcal{L}(Y \mid X)=\prod_{i=1}^{n} B\left(Y_{i} \mid X_{i}\right) .
$$

It follows that

$$
\mathcal{L}(Y)=\sum_{x \in \mathbb{N}^{n}} \mathbb{P}(X=x) \mathcal{L}(Y \mid X=x) .
$$

We will refer to $Y$ as a hidden Markov chain and to $X$ as its underlying Markov chain.

Stationary distributions and chains. The stationary distribution $\pi \in \mathbb{R}^{\mathbb{N}}$ of the Markov chain with transition kernel $A$ is the unique stochastic vector satisfying $A \pi=\pi$. The Markov chain induced by $\left(p_{1}, A\right)$ is said to be stationary if $p_{1}=\pi$. It is well known that, for ergodic Markov chains

$$
\pi=\lim _{n \rightarrow \infty} \mathcal{L}\left(X_{n}\right)=\lim _{n \rightarrow \infty} \mathbb{E} \hat{\pi}^{(n)},
$$

where

$$
\hat{\pi}_{x}^{(n)}=\frac{1}{n} \sum_{i=1}^{n} \mathbf{1}_{\left\{X_{i}=x\right\}}, \quad x \in \mathbb{N} .
$$


In the geometrically ergodic case, observing that $\mathbb{E} \hat{\pi}^{(n)}=(1 / n) \sum_{i=1}^{n} \mathcal{L}\left(X_{i}\right)$, we have

$$
\begin{aligned}
\left\|\mathbb{E} \hat{\pi}^{(n)}-\pi\right\|_{\mathrm{TV}} & =\left\|\frac{1}{n} \sum_{i=1}^{n}\left(\mathcal{L}\left(X_{i}\right)-\pi\right)\right\|_{\mathrm{TV}} \\
& \leq \frac{1}{n} \sum_{i=1}^{n}\left\|\mathcal{L}\left(X_{i}\right)-\pi\right\|_{\mathrm{TV}} \\
& =\frac{1}{n} \sum_{i=1}^{n}\left\|\sum_{x \in \mathbb{N}} \mathcal{L}\left(X_{i} \mid X_{1}=x\right) p_{1}(x)-\pi\right\|_{\mathrm{TV}} \\
& \leq \frac{1}{n} \sum_{i=1}^{n} \sum_{x \in \mathbb{N}} p_{1}(x)\left\|\mathcal{L}\left(X_{i} \mid X_{1}=x\right)-\pi\right\|_{\mathrm{TV}} \\
& \leq \frac{1}{n} \sum_{i=1}^{n} \sum_{x \in \mathbb{N}} p_{1}(x) G \theta^{i-1} \\
& =\frac{G}{(1-\theta) n} .
\end{aligned}
$$

For a hidden Markov chain, we define the stationary distribution $\rho=B \pi$, and observe that

$$
\rho=\lim _{n \rightarrow \infty} \mathcal{L}\left(Y_{n}\right)=\lim _{n \rightarrow \infty} \mathbb{E} \hat{\rho}^{(n)},
$$

where $\hat{\rho}^{(n)}$ is defined in (1.5). Since $\hat{\rho}^{(n)}$ is distributed as $B \hat{\pi}^{(n)}$, we have

$$
\left\|\mathbb{E} \hat{\rho}^{(n)}-\rho\right\|_{\mathrm{TV}} \leq\left\|\mathbb{E} \hat{\pi}^{(n)}-\pi\right\|_{\mathrm{TV}} \leq \frac{G}{(1-\theta) n} .
$$

The bound in (2.2) suggests that, at least to some degree, the statistical behavior of an HMM is controlled by its underlying Markov chain. We expand upon this observation further in the following lemma.

Lemma 2.1. Let $X$ and $X^{\prime}$ be two Markov chains induced by $(\xi, A)$ and $\left(\xi^{\prime}, A^{\prime}\right)$, respectively. For a given emission matrix $B$, let $Y$ and $Y^{\prime}$ be the hidden Markov chains induced by $(\xi, A, B)$ and $\left(\xi^{\prime}, A^{\prime}, B\right)$. Then

$$
\left\|\mathcal{L}\left(Y_{i \in I}\right)-\mathcal{L}\left(Y_{i \in I}^{\prime}\right)\right\|_{\mathrm{TV}} \leq\left\|\mathcal{L}\left(X_{i \in I}\right)-\mathcal{L}\left(X_{i \in I}^{\prime}\right)\right\|_{\mathrm{TV}}, \quad I \subseteq\{1, \ldots, n\}, n \in \mathbb{N}
$$

Proof. The proof is immediate from Jensen's inequality, since hidden Markov chains are convex mixtures of Markov chains.

The proofs of Theorems 1.2 and 1.3 will require bounds on $\left\|\hat{\rho}^{(n)}-\rho\right\|$, but unlike in (2.2), the expectation is on the outside of the norm.

\subsection{Markov contraction}

Let us recast the contraction coefficient defined in (1.3) in the language of Markov kernels

$$
\kappa=\sup _{x, x^{\prime} \in \mathbb{N}}\left\|A(\cdot \mid x)-A\left(\cdot \mid x^{\prime}\right)\right\|_{\mathrm{TV}} .
$$

The term 'contraction' is justified by the following simple fact [6], [21]. 
Lemma 2.2. (Markov [25].) For any two stochastic vectors $\xi, \psi \in \mathbb{R}^{\mathbb{N}}$, we have

$$
\|A(\xi-\psi)\|_{\mathrm{TV}} \leq \kappa\|\xi-\psi\|_{\mathrm{TV}} .
$$

Our principal application of this result will be in the context of geometrically ergodic Markov kernels.

Corollary 2.1. Let $A$ be a $(G, \theta)$-geometrically ergodic Markov kernel. Then, for all $n \in \mathbb{N}$, the $n$-step kernel $A^{n}$ has contraction coefficient $\kappa \leq 2 G \theta^{n}$.

Proof. Let $\pi$ be the stationary distribution of $A$ and $\xi, \psi \in \mathbb{R}^{\mathbb{N}}$ two point masses. Then

$$
\left\|A^{n} \xi-A^{n} \psi\right\|_{\mathrm{TV}} \leq\left\|A^{n} \xi-\pi\right\|_{\mathrm{TV}}+\left\|A^{n} \psi-\pi\right\|_{\mathrm{TV}} \leq 2 \tau_{n+1} \leq 2 G \theta^{n} .
$$

\subsection{Proof of main inequality}

In this section, we prove Theorem 1.1. The first order of business is to bound the $\eta$-mixing coefficient by the inverse mixing time, and, hence, in terms of $G$ and $\theta$.

Lemma 2.3. Let $Y$ be a $(G, \theta)$-geometrically ergodic hidden Markov chain and let $\bar{\eta}_{i j}$ and $\tau_{s}$ be as defined in (1.1) and (1.4), respectively. Then

$$
\bar{\eta}_{i j} \leq 2 \tau_{j-i+1} \leq 2 G \theta^{j-i}, \quad n \in \mathbb{N}, 1 \leq i<j \leq n .
$$

Proof. Let $X$ be the Markov chain underlying $Y$ and endow $\bar{\eta}_{i j}(X), \bar{\eta}_{i j}(Y)$ with the obvious meaning. Then [21, Theorem 7.1] shows that

$$
\bar{\eta}_{i j}(Y) \leq \bar{\eta}_{i j}(X)
$$

Next, Remark 4 and the theorem preceding it in [19] show that

$$
\bar{\eta}_{i j}(X) \leq \kappa\left(A^{j-i}\right)
$$

where $\kappa\left(A^{j-i}\right)$ is the contraction coefficient of the $(j-i)$-step Markov kernel of $X$. Finally, Corollary 2.1 yields

$$
\kappa\left(A^{j-i}\right) \leq 2 \tau_{j-i+1} \leq 2 G \theta^{j-i} .
$$

Proof of Theorem 1.1. By (1.2), it suffices to upper bound

$$
\|\Delta\|_{\infty}=1+\max _{1<i<n} \sum_{j=i+1}^{n} \bar{\eta}_{i j} .
$$

Applying Lemma 2.3, we obtain

$$
\max _{1<i<n} \sum_{j=i+1}^{n} \bar{\eta}_{i j} \leq 2 G \max _{1<i<n} \sum_{j=i+1}^{n} \theta^{j-i} \leq 2 G \sum_{k=1}^{\infty} \theta^{k} .
$$

Since $G \geq 1$ by assumption, we have

$$
1+2 G \sum_{k=1}^{\infty} \theta^{k} \leq 2 G \sum_{k=0}^{\infty} \theta^{k} \leq \frac{2 G}{1-\theta} .
$$




\subsection{Proof of the DKW-type inequality}

In this section we prove Theorem 1.2. Let $Y_{1}, Y_{2}, \ldots$ be a stationary $(G, \theta)$-geometrically ergodic hidden Markov chain with stationary distribution $\rho$, and define the $\{0,1\}$-indicator variables

$$
\xi_{i}^{(y)}=\mathbf{1}_{\left\{Y_{i}=y\right\}}, \quad i, y \in \mathbb{N} .
$$

Then $\hat{\rho}$, defined in (1.5), is given by $\hat{\rho}_{y}=(1 / n) \sum_{i=1}^{n} \xi_{i}{ }^{(y)}$, where we have dropped the superscript $(n)$ from $\hat{\rho}$ for readability. Observing that the map $\left(Y_{1}, \ldots, Y_{n}\right) \mapsto n\|\rho-\hat{\rho}\|_{\infty}$ is 1-Lipschitz under the Hamming metric (Lemma 2.7), we apply Theorem 1.1 to obtain

$$
\mathbb{P}\left(\|\rho-\hat{\rho}\|_{\infty}>\mathbb{E}\|\rho-\hat{\rho}\|_{\infty}+\varepsilon\right) \leq \exp \left(-\frac{n(1-\theta)^{2} \varepsilon^{2}}{2 G^{2}}\right) .
$$

Hence, it remains to bound $\mathbb{E}\|\rho-\hat{\rho}\|_{\infty}$.

Lemma 2.4. It holds that

$$
\mathbb{E}\|\rho-\hat{\rho}\|_{\infty} \leq \sqrt{\frac{1+2 G \theta}{n(1-\theta)}} .
$$

Remark. This estimate is nearly optimal: in the case where $Y_{i}$ are i.i.d. (i.e. $\theta=0$ ) Bernoulli variables with parameter $p$, we have [4, Theorem 1]

$$
\sqrt{\frac{p(1-p)}{2 n}} \leq \mathbb{E}\|\rho-\hat{\rho}\|_{\infty} \leq \sqrt{\frac{p(1-p)}{n}}, \quad n \geq 2, p \in\left(\frac{1}{n}, 1-\frac{1}{n}\right) .
$$

Proof. Jensen's inequality yields

$$
\begin{aligned}
\left(\mathbb{E}\|\rho-\hat{\rho}\|_{\infty}\right)^{2} & \leq \mathbb{E}\left[\|\rho-\hat{\rho}\|_{\infty}^{2}\right] \\
& \leq \mathbb{E}\left[\sum_{y \in \mathbb{N}}\left|\rho_{y}-\hat{\rho}_{y}\right|^{2}\right] \\
& =\sum_{y \in \mathbb{N}} \mathbb{E}\left(\rho_{y}-\hat{\rho}_{y}\right)^{2} \\
& =\sum_{y \in \mathbb{N}} \operatorname{var}\left[\hat{\rho}_{y}\right] .
\end{aligned}
$$

Putting $S_{n}^{(y)}=\sum_{i=1}^{n} \xi_{i}^{(y)}$, we have

$$
n^{2} \operatorname{var}\left[\hat{\rho}_{y}\right]=\mathbb{E}\left(S_{n}^{(y)}\right)^{2}-\left(\mathbb{E} S_{n}^{(y)}\right)^{2}
$$

and

$$
\mathbb{E} S_{n}^{(y)}=n \rho_{y}
$$

To bound $\mathbb{E}\left(S_{n}^{(y)}\right)^{2}$, we compute

$$
\begin{aligned}
\mathbb{E}\left(S_{n}^{(y)}\right)^{2} & =\mathbb{E}\left[\sum_{1 \leq i, j \leq n} \xi_{i}^{(y)} \xi_{j}^{(y)}\right] \\
& =\sum_{i=1}^{n} \mathbb{E}\left(\xi_{i}^{(y)}\right)^{2}+2 \sum_{1 \leq i<j \leq n} \mathbb{E}\left[\xi_{i}^{(y)} \xi_{j}^{(y)}\right] \\
& =n \rho_{y}+2 \sum_{1 \leq i<j \leq n} \mathbb{E}\left[\xi_{i}^{(y)} \xi_{j}^{(y)}\right],
\end{aligned}
$$


where the last identity holds since $\xi_{i}^{(y)} \in\{0,1\}$. It now remains to estimate $\mathbb{E}\left[\xi_{i}^{(y)} \xi_{j}^{(y)}\right]$. To this end, we claim that

$$
\left\|\mathcal{L}\left(Y_{i} \mid Y_{1}=y\right)-\rho\right\|_{\infty} \leq G \theta^{i-1}, \quad i, y \in \mathbb{N}
$$

Indeed, denoting the parameters of $Y$ by $(\pi, A, B)$ and letting $X$ be the underlying Markov chain, we have

$$
\begin{aligned}
\left\|\mathcal{L}\left(Y_{i} \mid Y_{1}=y_{1}\right)-\rho\right\|_{\infty} & \leq\left\|\mathcal{L}\left(Y_{i} \mid Y_{1}=y_{1}\right)-\rho\right\|_{\mathrm{TV}} \\
& =\frac{1}{2} \sum_{y_{i} \in \mathbb{N}}\left|\mathbb{P}\left(Y_{i}=y_{i} \mid Y_{1}=y_{1}\right)-\rho_{y_{i}}\right| \\
& =\frac{1}{2} \sum_{y_{i} \in \mathbb{N}}\left|\sum_{x_{i} \in \mathbb{N}} B_{y_{i}, x_{i}}\left(\mathbb{P}\left(X_{i}=x_{i} \mid Y_{1}=y_{1}\right)-\pi_{x_{i}}\right)\right| \\
& \leq \frac{1}{2} \sum_{y_{i} \in \mathbb{N}} \sum_{x_{i} \in \mathbb{N}} B_{y_{i}, x_{i}}\left|\mathbb{P}\left(X_{i}=x_{i} \mid Y_{1}=y_{1}\right)-\pi_{x_{i}}\right| \\
& =\frac{1}{2} \sum_{x_{i} \in \mathbb{N}}\left|\mathbb{P}\left(X_{i}=x_{i} \mid Y_{1}=y_{1}\right)-\pi_{x_{i}}\right| \\
& =\left\|\sum_{x_{1} \in \mathbb{N}} \mathcal{L}\left(X_{i} \mid X_{1}=x_{1}\right) \mathbb{P}\left(X_{1}=x_{1} \mid Y_{1}=y_{1}\right)-\pi\right\|_{\mathrm{TV}} \\
& \leq \sup _{x_{1} \in \mathbb{N}}\left\|\mathcal{L}\left(X_{i} \mid X_{1}=x_{1}\right)-\pi\right\|_{\mathrm{TV}} \\
& \leq G \theta^{i-1} .
\end{aligned}
$$

Hence,

$$
\begin{aligned}
\mathbb{E}\left[\xi_{i}^{(y)} \xi_{j}^{(y)}\right] & =\mathbb{P}\left(Y_{i}=y, Y_{j}=y\right) \\
& =\mathbb{P}\left(Y_{1}=y, Y_{j-i+1}=y\right) \\
& =\mathbb{P}\left(Y_{1}=y\right) \mathbb{P}\left(Y_{j-i+1}=y \mid Y_{1}=y\right) \\
& \leq \rho_{y}\left(\rho_{y}+G \theta^{j-i}\right)
\end{aligned}
$$

and, therefore,

$$
\begin{aligned}
\sum_{1 \leq i<j \leq n} \mathbb{E}\left[\xi_{i}^{(y)} \xi_{j}^{(y)}\right] & =\sum_{k=1}^{n-1}(n-k) \mathbb{P}\left(Y_{1}=y\right) \mathbb{P}\left(Y_{k+1}=y \mid Y_{1}=y\right) \\
& \leq \sum_{k=1}^{n-1}(n-k) \rho_{y}\left(\rho_{y}+G \theta^{k}\right) \\
& =\frac{n(n-1)}{2} \rho_{y}^{2}+\frac{G \theta}{1-\theta}\left(n-\frac{1-\theta^{n}}{1-\theta}\right) \rho_{y} \\
& \leq \frac{n(n-1)}{2} \rho_{y}^{2}+n \frac{G \theta}{1-\theta} \rho_{y} .
\end{aligned}
$$


Combining (2.5), (2.6), (2.7), and (2.9), we have

$$
\begin{aligned}
\operatorname{var}\left[\hat{\rho}_{y}\right] & \leq \frac{1}{n^{2}}\left(n \rho_{y}+n(n-1) \rho_{y}^{2}+2 n \frac{G \theta}{1-\theta} \rho_{y}-n^{2} \rho_{y}^{2}\right) \\
& =\frac{\rho_{y}}{n}\left(1-\rho_{y}+\frac{2 G \theta}{1-\theta}\right) \\
& \leq \rho_{y} \frac{1+2 G \theta}{n(1-\theta)} .
\end{aligned}
$$

Since $\sum_{y \in \mathbb{N}} \rho_{y}=1$, the claim follows from (2.4).

Remark. Note that in the process of proving a deviation estimate on $\|\rho-\hat{\rho}\|_{\infty}$, we have actually proven a stronger one: namely, for the $\ell_{2}$ norm.

\subsection{Proof of the uniform Chernoff bound}

In this section we prove Theorem 1.3. As before, $Y_{1}, Y_{2}, \ldots$ is a stationary $(G, \theta)$-geometrically ergodic hidden Markov chain with stationary distribution $\rho$. Since by Lemma 2.7 the map $\left(Y_{1}, \ldots, Y_{n}\right) \mapsto n\|\rho-\hat{\rho}\|_{\mathrm{TV}}$ is 1-Lipschitz under the Hamming metric, Theorem 1.1 applies

$$
\mathbb{P}\left(\|\rho-\hat{\rho}\|_{\mathrm{TV}}>\mathbb{E}\|\rho-\hat{\rho}\|_{\mathrm{TV}}+\varepsilon\right) \leq \exp \left(-\frac{n(1-\theta)^{2} \varepsilon^{2}}{2 G^{2}}\right) .
$$

As before, the crux of the matter is to bound $\mathbb{E}\|\rho-\hat{\rho}\|_{\mathrm{TV}}$. Recall the definition of $\Lambda_{n}$ from the statement of Theorem 1.3.

Lemma 2.5. It holds that

$$
\mathbb{E}\|\rho-\hat{\rho}\|_{\mathrm{TV}} \leq \Lambda_{n}
$$

Remark. This bound is nearly optimal: when the $Y_{i}$ are i.i.d., we have [4, Proposition 3]

$$
\mathbb{E}\|\rho-\hat{\rho}\|_{\mathrm{TV}} \geq \frac{1}{4} \Lambda_{n}-\frac{1}{8 \sqrt{n}}, \quad n \geq 2, p \in\left(\frac{1}{n}, 1-\frac{1}{n}\right) .
$$

Proof. We proceed by breaking up the expectation into two terms

$$
\mathbb{E}\|\rho-\hat{\rho}\|_{\mathrm{TV}}=\frac{1}{2} \sum_{y: \rho_{y}<1 / n} \mathbb{E}\left|\rho_{y}-\hat{\rho}_{y}\right|+\frac{1}{2} \sum_{y: \rho_{y} \geq 1 / n} \mathbb{E}\left|\rho_{y}-\hat{\rho}_{y}\right|,
$$

and bounding each term separately. To bound the second term, we note, as in the proof of Lemma 2.4, that

$$
\mathbb{E}\left|\rho_{y}-\hat{\rho}_{y}\right| \leq \sqrt{\operatorname{var}\left[\hat{\rho}_{y}\right]} \leq \sqrt{\rho_{y} \frac{1+2 G \theta}{n(1-\theta)}}, \quad y \in \mathbb{N}
$$

To bound the first term, we recall the indicator variables $\xi_{i}^{(y)}$ defined in (2.3) such that

$$
n \mathbb{E}\left|\rho_{y}-\hat{\rho}_{y}\right|=\mathbb{E}\left|\sum_{i=1}^{n} \xi_{i}^{(y)}-n \rho_{y}\right| \leq n \mathbb{E}\left|\xi_{i}^{(y)}-\rho_{y}\right|=2 n \rho_{y}\left(1-\rho_{y}\right) \leq 2 n \rho_{y},
$$

where stationarity was used in the last line of the derivation.

Combining the last display with (2.11) and (2.12) yields the claim. 
Proof of Theorem 1.3. (a) Since obviously

$$
\sum_{\rho_{y}<1 / n} \rho_{y} \rightarrow 0 \quad \text { as } n \rightarrow \infty,
$$

it suffices to show that

$$
\frac{1}{\sqrt{n}} \sum_{\rho_{y} \geq 1 / n} \sqrt{\rho_{y}} \rightarrow 0 \quad \text { as } n \rightarrow \infty .
$$

The latter was proved in [4, Lemma 7], but we will present a simpler proof here. This elegant proof is due to Asaf Shachar. Andrew Barron points out that (2.13) may be easily derived from Lebesgue's dominated convergence theorem.

Assume without loss of generality that $\rho_{1} \geq \rho_{2} \geq \cdots$, pick an arbitrary $\varepsilon>0$, and let $N \in \mathbb{N}$ be large enough so that $\sum_{j \geq N} \rho_{j}<\varepsilon$.

Then

$$
\begin{aligned}
\frac{1}{\sqrt{n}} \sum_{\rho_{j} \geq 1 / n} \sqrt{\rho_{j}} & \leq \frac{1}{\sqrt{n}} \sum_{j \leq N} \sqrt{\rho_{j}}+\frac{1}{\sqrt{n}} \sum_{j>N, \rho_{j} \geq 1 / n} \sqrt{\rho_{j}} \\
& \leq \sqrt{\frac{N}{n}}+\frac{1}{\sqrt{n}} \sqrt{\sum_{\rho_{j} \geq 1 / n} 1} \sqrt{\sum_{j>N} \rho_{j}} \\
& \leq \sqrt{\frac{N}{n}}+\sqrt{\varepsilon}
\end{aligned}
$$

since there can be at most $n$ terms with $\rho_{j} \geq 1 / n$.

(b) The claim follows from (2.10) and the fact that for any two distributions $\phi, \psi \in \mathbb{R}^{\mathbb{N}}$,

$$
\|\phi-\psi\|_{\mathrm{TV}}=\sup _{E \subseteq \mathbb{N}}|\phi(E)-\psi(E)| .
$$

\subsection{The stationarity assumption}

To state the bounds in Theorems 1.2 and 1.3 more cleanly, we had assumed that the Markov and hidden Markov chains in question are stationary, i.e. that the initial distribution $p_{1}$ is identical to the stationary one $\pi$. In this section we show (Corollary 2.2) that for strongly mixing chains the stationarity assumption may be relaxed, at the cost of additional terms in the deviation bounds.

Let $Y=\left(Y_{1}, \ldots, Y_{n}\right)$ be a $(G, \theta)$-geometrically ergodic hidden Markov chain with parameters $\left(\pi^{\prime}, A, B\right)$, where $\pi^{\prime} \in \mathbb{R}^{\mathbb{N}}$ is some stochastic vector. A simple dimension-free bound on the statistical distance between $Y$ and its stationary version is available.

Theorem 2.1. Let $Y^{\prime}=\left(Y_{1}^{\prime}, \ldots, Y_{n}^{\prime}\right)$ be the stationary version of $Y$, i.e. a HMM with parameters $(\pi, A, B)$, where $\pi$ is the stationary distribution of the kernel A. Then

$$
\left\|\mathcal{L}(Y)-\mathcal{L}\left(Y^{\prime}\right)\right\|_{\mathrm{TV}} \leq\left\|\pi-\pi^{\prime}\right\|_{\mathrm{TV}} .
$$

First, we prove an analogous result for Markov chains.

Lemma 2.6. Let $A$ be a Markov kernel and let $\xi, \xi^{\prime} \in \mathbb{R}^{\mathbb{N}}$ be two arbitrary stochastic vectors. Let $X=\left(X_{1}, \ldots, X_{n}\right)$ and $X^{\prime}=\left(X_{1}^{\prime}, \ldots, X_{n}^{\prime}\right)$ be the Markov chains induced by $(\xi, A)$ and $\left(\xi^{\prime}, A\right)$, respectively. Then

$$
\left\|\mathcal{L}(X)-\mathcal{L}\left(X^{\prime}\right)\right\|_{\mathrm{TV}}=\left\|\xi-\xi^{\prime}\right\|_{\mathrm{TV}}
$$


Proof. We have

$$
\begin{aligned}
\left\|\mathcal{L}(X)-\mathcal{L}\left(X^{\prime}\right)\right\|_{\mathrm{TV}} & =\frac{1}{2} \sum_{x \in \mathbb{N}^{n}}\left|\left(\xi_{x_{1}}-\xi_{x_{1}}^{\prime}\right) A_{x_{2}, x_{1}} \cdots A_{x_{n}, x_{n-1}}\right| \\
& =\frac{1}{2} \sum_{x \in \mathbb{N}^{n}} A_{x_{2}, x_{1}} \cdots A_{x_{n}, x_{n-1}}\left|\xi_{x_{1}}-\xi_{x_{1}}^{\prime}\right| \\
& =\frac{1}{2} \sum_{x_{1} \in \mathbb{N}}\left|\xi_{x_{1}}-\xi_{x_{1}}^{\prime}\right| \\
& =\left\|\xi-\xi^{\prime}\right\|_{\mathrm{TV}} .
\end{aligned}
$$

Proof of Theorem 2.1. Lemma 2.1 lets us restrict our attention to the underlying Markov chains $X$ and $X^{\prime}$, respectively

$$
\begin{aligned}
\left\|\mathcal{L}\left(Y_{1 \leq i \leq n}\right)-\mathcal{L}\left(Y_{1 \leq i \leq n}^{\prime}\right)\right\|_{\mathrm{TV}} & \leq\left\|\mathcal{L}\left(X_{1 \leq i \leq n}\right)-\mathcal{L}\left(X_{1 \leq i \leq n}^{\prime}\right)\right\|_{\mathrm{TV}} \\
& =\left\|\mathcal{L}\left(X_{1}\right)-\mathcal{L}\left(X_{1}^{\prime}\right)\right\|_{\mathrm{TV}} \\
& =\left\|\pi-\pi^{\prime}\right\|_{\mathrm{TV}},
\end{aligned}
$$

where the first identity follows from Lemma 2.6.

Corollary 2.2. Let $Y_{1}, Y_{2}, \ldots$ be a (not necessarily stationary) $\mathbb{N}$-valued $(G, \theta)$-geometrically ergodic hidden Markov chain with stationary distribution $\rho=B \pi$ and initial distribution $\rho^{\prime}=B \pi^{\prime}$. Then the deviation bounds stated in Theorems 1.2 and 1.3 hold with an additive correction of $\left\|\pi-\pi^{\prime}\right\|_{\mathrm{TV}}$ on the right-hand side.

\subsection{Auxiliary lemma}

The Hamming metric on $\mathbb{N}^{n}$ is defined by $d(\boldsymbol{x}, \boldsymbol{y})=\sum_{i=1}^{n} \mathbf{1}_{\left\{x_{i} \neq y_{i}\right\}}$ for $\boldsymbol{x}, \boldsymbol{y} \in \mathbb{N}^{n}$.

Lemma 2.7. Suppose $n \in \mathbb{N}$ and $\boldsymbol{p} \in \mathbb{R}^{\mathbb{N}}$ is a distribution. Define the functions $g, h: \mathbb{N}^{n} \rightarrow \mathbb{R}$

$$
\begin{array}{ll}
g(\boldsymbol{x})=\sup _{j \in \mathbb{N}}\left|n p_{j}-\sum_{i=1}^{n} \mathbf{1}_{\left\{x_{i}=j\right\}}\right|, & \boldsymbol{x} \in \mathbb{N}^{n}, \\
h(\boldsymbol{x})=\sum_{j \in \mathbb{N}}\left|n p_{j}-\sum_{i=1}^{n} \mathbf{1}_{\left\{x_{i}=j\right\}}\right|, & \boldsymbol{x} \in \mathbb{N}^{n} .
\end{array}
$$

Then $\|g\|_{\text {Lip }} \leq 1$ and $\|h\|_{\text {Lip }} \leq 2$ with respect to the Hamming metric

$$
|g(\boldsymbol{x})-g(\boldsymbol{y})| \leq d(\boldsymbol{x}, \boldsymbol{y}), \quad|h(\boldsymbol{x})-h(\boldsymbol{y})| \leq 2 d(\boldsymbol{x}, \boldsymbol{y}) \quad \text { for all } \boldsymbol{x}, \boldsymbol{y} \in \mathbb{N}^{n} .
$$

Proof. We only prove the claim for $h$ (the proof for $g$ is analogous). Let the function $\hat{n}_{j}: \mathbb{N}^{n} \rightarrow \mathbb{N}$ count the number of times $j$ appears in $x$; formally, $\hat{n}_{j}(x)=\sum_{i=1}^{n} \mathbf{1}_{\left\{x_{i}=j\right\}}$. Now suppose $\boldsymbol{x}, \boldsymbol{y} \in \mathbb{N}^{n}$ differ only in coordinate $k$, with $x_{k}=a$ and $y_{k}=b$. Then

$$
\begin{aligned}
h(\boldsymbol{x}) & -h(\boldsymbol{y}) \\
& =\sum_{j \in \mathbb{N}}\left|n p_{j}-\hat{n}_{j}(\boldsymbol{x})\right|-\sum_{j \in \mathbb{N}}\left|n p_{j}-\hat{n}_{j}(\boldsymbol{y})\right| \\
& =\left(\left|n p_{a}-\hat{n}_{a}(\boldsymbol{x})\right|+\left|n p_{b}-\hat{n}_{b}(\boldsymbol{x})\right|\right)-\left(\left|n p_{a}-\hat{n}_{a}(\boldsymbol{y})\right|+\left|n p_{b}-\hat{n}_{b}(\boldsymbol{y})\right|\right) \\
& =\left(\left|n p_{a}-\hat{n}_{a}(\boldsymbol{x})\right|+\left|n p_{b}-\hat{n}_{b}(\boldsymbol{x})\right|\right)-\left(\left|n p_{a}-\left(\hat{n}_{a}(\boldsymbol{x})-1\right)\right|+\left|n p_{b}-\left(\hat{n}_{b}(\boldsymbol{x})+1\right)\right|\right) \\
& \leq\left|n p_{a}-\hat{n}_{a}(\boldsymbol{x})\right|-\left|n p_{a}-\left(\hat{n}_{a}(\boldsymbol{x})-1\right)\right||+|\left|n p_{b}-\hat{n}_{b}(\boldsymbol{x})\right|-\left|n p_{b}-\left(\hat{n}_{b}(\boldsymbol{x})+1\right)\right| \mid \\
& \leq 2 .
\end{aligned}
$$




\section{Acknowledgements}

We thank the anonymous referee for carefully reading the manuscript and offering helpful suggestions. This research was partially supported by the Israel Science Foundation (grant number 1141/12) and a Yahoo Faculty award.

\section{References}

[1] AdamczaK, R. (2008). A tail inequality for suprema of unbounded empirical processes with applications to Markov chains. Electron. J. Prob. 13, 1000-1034.

[2] AdamczaK, R. and Bednorz, W. (2012). Exponential concentration inequalities for additive functionals of Markov chains. Preprint. Available at http://arxiv.org/abs/1201.3569v1.

[3] Anandkumar, A., Hsu, D. and Kakade, S. M. (2012). A method of moments for mixture models and hidden Markov models. In Proc. 25th Annual Conf. Learning Theory (Edinburgh, June 2012), 34 pp.

[4] Berend, D. And Kontorovich, A. (2013). A sharp estimate of the binomial mean absolute deviation with applications. Statist. Prob. Lett. 83, 1254-1259.

[5] BoвKov, S. G. AND Götze, F. (2010). Concentration of empirical distribution functions with applications to non-i.i.d. models. Bernoulli 16, 1385-1414.

[6] Brémaud, P. (1999). Markov Chains: Gibbs Fields, Monte Carlo Simulation, and Queues. Springer, New York.

[7] Chazottes, J.-R. And Redig, F. (2009). Concentration inequalities for Markov processes via coupling. Electron. J. Prob. 14, 1162-1180.

[8] Chazottes, J.-R., Collet, P., Külske, C. and Redig, F. (2007). Concentration inequalities for random fields via coupling. Prob. Theory Relat. Fields 137, 201-225.

[9] Chung, K.-M., Lam, H., Liu, Z. and Mitzenmacher, M. (2012). Chernoff-Hoeffding bounds for Markov chains: generalized and simplified. In 29th Internat. Symp. Theoret. Aspects Comput. Sci., Schloss Dagstuhl, Wadern, pp. 124-135.

[10] Diaconis, P. and Saloff-Coste, L. (1996). Logarithmic Sobolev inequalities for finite Markov chains. Ann. Appl. Prob. 6, 695-750.

[11] Diaconis, P. And Saloff-Coste, L. (1996). Nash inequalities for finite Markov chains. J. Theoret. Prob. 9, 459-510.

[12] Dinwoodie, I. H. (1995). A probability inequality for the occupation measure of a reversible Markov chain. Ann. Appl. Prob. 5, 37-43.

[13] Dinwoodie, I. H. (1998). Expectations for nonreversible Markov chains. J. Math. Anal. Appl. 220, 585-596.

[14] DvoretzKy, A., Kiefer, J. And Wolfowitz, J. (1956). Asymptotic minimax character of the sample distribution function and of the classical multinomial estimator. Ann. Math. Statist. 27, 642-669.

[15] FILL, J. A. (1991). Eigenvalue bounds on convergence to stationarity for nonreversible Markov chains, with an application to the exclusion process. Ann. Appl. Prob. 1, 62-87.

[16] Gillman, D. (1998). A Chernoff bound for random walks on expander graphs. SIAM J. Comput. 27, 1203-1220.

[17] Hsu, D., KaKade, S. M. AND Zhang, T. (2009). A spectral algorithm for learning hidden Markov models. In Proc. 22nd Annual Conf. Learning Theory (Montreal, June 2009), 10 pp.

[18] Kahale, N. (1997). Large deviation bounds for Markov chains. Combin. Prob. Comput. 6, $465-474$.

[19] Kontorovich, A. (2012). Obtaining measure concentration from Markov contraction. Markov Process. Relat. Fields 18, 613-638.

[20] Kontorovich, A., Nadler, B. and Weiss, R. (2013). On learning parametric-output HMMS. In Proc. 30th Internat. Conf. Machine Learning (June 2013, Atlanta), pp. 702-710.

[21] Kontorovich, L. and Ramanan, K. (2008). Concentration inequalities for dependent random variables via the martingale method. Ann. Prob. 36, 2126-2158.

[22] Kontoyiannis, I. And Meyn, S. P. (2012). Geometric ergodicity and the spectral gap of non-reversible Markov chains. Prob. Theory Relat. Fields 154, 327-339.

[23] León, C. A. and Perron, F. (2004). Optimal Hoeffding bounds for discrete reversible Markov chains. Ann. Appl. Prob. 14, 958-970.

[24] Lezaud, P. (1998). Chernoff-type bound for finite Markov chains. Ann. Appl. Prob. 8, 849-867.

[25] Markov, A. A. (1906). Extension of the law of large numbers to dependent quantities. Izvestiia Fiz.-Matem. Obsch. Kazan Univ. 15, 135-156.

[26] Marton, K. (1996). Bounding $\bar{d}$-distance by informational divergence: a method to prove measure concentration. Ann. Prob. 24, 857-866.

[27] Marton, K. (1998). Measure concentration for a class of random processes. Prob. Theory Relat. Fields 110, 427-439.

[28] Marton, K. (2003). Measure concentration and strong mixing. Studia Sci. Math. Hungarica 40, 95-113. 
[29] Marton, K. (2004). Measure concentration for Euclidean distance in the case of dependent random variables. Ann. Prob. 32, 2526-2544.

[30] Massart, P. (1990). The tight constant in the Dvoretzky-Kiefer-Wolfowitz inequality. Ann. Prob. 18, 1269-1283.

[31] Mossel, E. And Roch, S. (2006). Learning nonsingular phylogenies and hidden Markov models. Ann. Appl. Prob. 16, 583-614.

[32] Rio, E. (2000). Inégalités de Hoeffding pour les fonctions lipschitziennes de suites dépendantes. C. R. Acad. Sci. Paris Sér. I Math. 330, 905-908.

[33] SAMson, P.-M. (2000). Concentration of measure inequalities for Markov chains and $\Phi$-mixing processes. Ann. Prob. 28, 416-461.

[34] SiddiQI, S., Boots, B. And Gordon, G. (2010). Reduced-rank hidden Markov models. In Proc. 13th Internat Conf. Artificial Intelligence Statist. (Sardinia, Italy, May 2010), pp. 741-748.

[35] Wagner, R. (2008). Tail estimates for sums of variables sampled by a random walk. Combin. Prob. Comput. 17, 307-316.

[36] Zou, J. Y., Hsu, D., Parkes, D. and Adams, R. P. (2013). Contrastive learning using spectral methods. In Advances in Neural Information Processing Systems 26, eds C. J. C. Burges et al., pp. 2238-2246. 